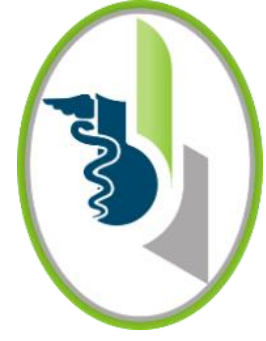

ACCESO $\odot$ ABIERTO

Para citaciones: Marrugo, A., Mendoza, L., Payares, S. (2021). Hallazgos macroscópicos y microscópicos en el sistema respiratorio en autopsias de pacientes con covid-19. Revista Ciencias Biomédicas, 10(3), 172-188. https://doi.org/10.32997/rcb-2021-3334

Recibido: 10 de mayo de 2021 Aprobado: 15 de junio de 2021

Autor de correspondencia: Laura Marcela Mendoza Carmona lmendozac@unicartagena.edu.co

Editor: Inés Benedetti. Universidad de Cartagena-Colombia.

Copyright: (C) 2021. Marrugo, A., Mendoza, L., Payares, S. Este es un artículo de acceso abierto, distribuido bajo los términos de la licencia https://creativecommons.org/licenses/by-nc-

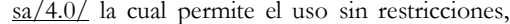
distribución y reproducción en cualquier medio, siempre y cuando el original, el autor y la fuente sean acreditados.

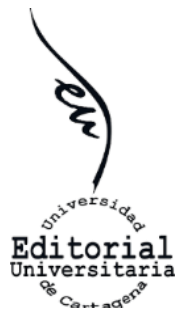

\section{Hallazgos macroscópicos y microscópicos en el sistema respiratorio en autopsias de pacientes con covid-19}

\author{
Macroscopic and microscopic findings in the respiratory system \\ in patients with covid-19
}

Ariel Camilo Marrugo Ortiz ${ }^{(i D}$, Laura Marcela Mendoza Carmona ${ }^{1}$ (D), Sebastián David Payares Flórez ${ }^{1}$ (D)

${ }^{1}$ Pregrado, Facultad de Medicina, Universidad de Cartagena, Cartagena, Colombia.

\section{RESUMEN}

Introducción: desde finales del 2019, el mundo se ha visto hostigado por la aparición de un nuevo coronavirus (SARS-CoV-2) en Wuhan, China, que se extendió globalmente y es el agente etiológico de la enfermedad conocida como COVID-19. Esta enfermedad tiene tres etapas consecutivas de gravedad y está asociada con el desarrollo del síndrome de dificultad respiratoria aguda (SDRA).

Objetivo: describir las principales lesiones pulmonares, macroscópicas y microscópicas, asociadas con la infección por SARS-CoV-2, con base en hallazgos de autopsias realizadas a pacientes fallecidos por Covid-19.

Métodos: se realizó una búsqueda en las bases de datos PubMed, Google Scholar, Scielo, Clinical Key y MedRxiv que incluyó artículos publicados entre el 1 de enero del 2020 y el 31 de diciembre de 2020.

Resultados: el principal hallazgo macroscópico descrito fue la presencia de pulmones pesados además de superficies con áreas azul-rojizas. Se encontró en algunos casos traqueo-bronquitis y tráqueas levemente eritematosas. En hallazgos microscópicos el patrón de lesión pulmonar más común fue el daño alveolar difuso (DAD) presentado en el $88 \%$ de los casos. Se encontraron cambios en los capilares constituyendo 3 características histológicas: Lesión endotelial, alteración de las membranas de células endoteliales y angiogénesis intususceptiva.

Conclusión: la gravedad de los hallazgos está determinada por la edad del paciente y el padecimiento de diversas comorbilidades/afecciones sobre todo relacionadas con el sistema respiratorio y cardiovascular, estos factores influyen en el desarrollo de hallazgos heterogéneos tanto macroscópicos como microscópicos.

Palabras Clave: SARS-COV-2; COVID-19; epitelio respiratorio; histopatología; pulmón; daño alveolar difuso; síndrome de dificultad respiratoria aguda. 


\begin{abstract}
Introduction: since the end of 2019, the world has been harassed by the appearance of a new coronavirus (SARS-CoV-2) in Wuhan, China, which spread globally and is the etiological agent of the disease known as COVID-19. This disease has three consecutive stages of severity and is associated with the development of acute respiratory distress syndrome (ARDS).
\end{abstract}

Objective: to describe the main macroscopic and microscopic lung lesions associated with SARS-CoV-2 infection, based on autopsy findings on patients who died from Covid-19.

Methods: a search was carried out in the PubMed, Google Scholar, Scielo, Clinical Key and MedRxiv databases that included articles published between January 1, 2020 and December 31, 2020.

Results: the main macroscopic finding described was the presence of heavy lungs as well as surfaces with blue-reddish areas. Tracheo-bronchitis and slightly erythematous tracheas were found in some cases. In microscopic findings, the most common pattern of lung injury was diffuse alveolar damage (DAD), presented in $88 \%$ of cases. Changes were found in the capillaries constituting 3 histological characteristics: endothelial lesion, alteration of endothelial cell membranes and intussusceptive angiogenesis.

Conclusions: the severity of findings is determined by the age of the patient and the suffering of various comorbidities / conditions especially related to the respiratory and cardiovascular system, these factors influence the development of heterogeneous macroscopic and microscopic findings.

Keywords: SARS-COV-2; COVID-19; respiratory epithelium; histopathology; lung; diffuse alveolar damage; acute respiratory distress syndrome.

\section{INTRODUCCIÓN}

Desde finales del año 2019, el mundo se ha visto hostigado por la aparición de un nuevo coronavirus de tipo 2 causante del síndrome respiratorio agudo severo (SARS-CoV-2) en Wuhan, China, el cual es el agente etiológico de la enfermedad que se conoce como COVID-19 (Co de "Coronavirus", Vi de "Virus", D del inglés "Disease que significa enfermedad" y 19 porque fue descubierto en el año 2019), que se extendió hasta convertirse en una pandemia(1)(2)(3). Los coronavirus son virus de ARN monocatenarios positivos, con envoltura, que causan predominantemente infecciones en el tracto respiratorio. Los brotes anteriores causados por coronavirus incluyen el síndrome respiratorio agudo severo (SARS) ocurrido en el año 2003 y el síndrome respiratorio de oriente medio (MERS) en el año 2012. Ambos coronavirus se originaron en murciélagos que infectaron huéspedes secundarios como los gatos de algalia (SARS) y los camellos dromedarios (MERS) (4).

La estructura del SARS-CoV-2 está compuesta por una nucleocápside que se encarga de proteger el material genético viral y una envoltura externa. El material genético viral está asociado a la proteína de la nucleocápside $(\mathrm{N})$, una proteína fosforilada que ayuda en el plegamiento apropiado del ARN genómico a la nucleocápside (5). El genoma tiene un tamaño de 29.891 nucleótidos, puede codificar 9.860 aminoácidos y codifica diversas proteínas estructurales indispensables para la supervivencia del virus. 
Entre ellas, la proteína espiga también conocida como Spike (S), la de envoltura (E), la de membrana (M), y la nucleoproteína anteriormente mencionada (N); estas, aunque no son las únicas proteínas codificables, se resaltan debido a su papel en la patogenia vírica (1).

El SARS-Cov-2 infecta a las células humanas a través de la unión al receptor de la enzima convertidora de angiotensina 2 (ACE2h, del inglés: human angiotensin-converting enzyme 2) expresada en gran medida en las células epiteliales del tracto respiratorio, en células endoteliales y en otros tipos celulares. La glicoproteína Spike (S) que se encuentra en la superficie del virus es la que permite la unión al ACE2h, su escisión por las proteasas de la célula huésped facilita la fusión de las membranas vírica y celular, y permite el ingreso del virus (6). El SARS-Cov-2 es altamente contagioso en comparación a los otros coronavirus humanos que se autolimitan a infecciones en las vías respiratorias superiores y ocasionalmente en el tracto inferior en pacientes inmunodeprimidos y ancianos (7).

En general, la Covid-19 tiene tres etapas consecutivas de gravedad. La primera es la infección por SARS-CoV-2. En esta etapa, se suelen desarrollar síntomas parecidos a los de la gripe, debido en gran parte a la propia infección viral, aunque algunos pacientes pueden llegar a desarrollar neumonía viral y requerir hospitalización e incluso respiración mecánica. La segunda etapa está relacionada con la inflamación pulmonar y las coagulopatías. Estas pueden desarrollarse una tras otra, pero tienden a superponerse. Además, hay un aumento significativo de biomarcadores inflamatorios (PCR, ferritina, IL-6, IL-1 y dímero D) que se asocia con el desarrollo del síndrome de dificultad respiratoria aguda (SDRA) y una evolución clínica desfavorable. Y la última etapa se caracteriza por fibrosis a nivel pulmonar (3).

El SARS-CoV-2 produce también manifestaciones extrapulmonares, como diarrea, linfopenia, y, sobre todo en casos graves, pueden verse afectados el tracto intestinal, el hígado, los riñones y presentarse un síndrome de disfunción multiorgánica, generalmente acompañado de una tormenta de citoquinas que puede ser letal, especialmente en paciente inmunodeprimidos y de la tercera edad (2). La COVID-19 entonces parece ser menos mortal en pacientes jóvenes con pocas o ninguna comorbilidad, tales como hipertensión, diabetes mellitus, enfermedad pulmonar obstructiva crónica (EPOC), tabaquismo, infección por el virus de la inmunodeficiencia humana (VIH), inmunosupresión y antecedentes de trasplantes. Sin embargo, los pacientes mayores que presentan dichas comorbilidades tienden a morir en las primeras etapas de la enfermedad (8).

Entre los factores que influyen en la alta contagiosidad del SARS-CoV-2 se encuentran el gran número de pacientes asintomáticos y el desconocimiento de la enfermedad los que en conjunto contribuyeron a su rápida difusión por todo el mundo debido a que los pacientes asintomáticos pueden contagiar el virus, sin embargo, no está claro durante cuánto tiempo y como afecta la historia natural de la enfermedad (1).

En el presente estudio se describen los diferentes hallazgos macroscópicos y microscópicos en autopsias realizadas a pacientes fallecidos por COVID-19, enfocados en las vías respiratorias superiores e inferiores con el objetivo de informar las principales lesiones pulmonares microscópicas y macroscópicas en la infección por SARS-CoV-2.

\section{MÉTODOS}

Se llevó a cabo una revisión sistemática utilizando los siguientes términos de búsqueda: SARS-CoV-2, COVID-19, histopathology, pathology, lung, en las bases de datos, PubMed, Google Scholar, Scielo, Clinical Key y MedRxiv. La búsqueda incluyó artículos publicados entre el 1 de enero de 2020 y el 31 de diciembre de 2020. Además, se realizó una búsqueda manual en las referencias de los estudios más relevantes para identificar artículos citados que no hubiesen sido incluidos en la búsqueda inicial. Dicha búsqueda arrojó como resultado 465 artículos, a los que se les aplicaron los siguientes criterios de selección: inclusión de informes histopatológicos de 
autopsias o casos post mortem de COVID-19, sospecha clínica de COVID-19 como principal causa de muerte incluyéndose solo autopsias, descripción de al menos un caso, confirmación de infección por SARS-COV-2 mediante la prueba RT-PCR, y descripción histológica suficiente de cada caso informado en el estudio; quedando en la selección final los 51 estudios incluidos en la revisión.

Se identificaron las características de cada estudio incluido, a saber, datos demográficos de la población de estudio como edad y sexo, hallazgos macroscópicos y microscópicos específicos en los órganos del sistema respiratorio y las limitaciones descritas. Se realizaron análisis exhaustivos de los patrones para la realización de las tablas, donde también se tuvieron en cuenta datos de resultados de ensayos de inmunohistoquímica e inmunofluorescencia.

\section{RESULTADOS}

Se llevó a cabo una revisión sistemática de 51 artículos que describen en total 576 pacientes presentados en 39 series de casos y 12 reportes de casos individuales. Los estudios revisados incluyeron pacientes de todo género cuya edad media estuvo entre 64 y 65 años, algunos de los cuales presentaban comorbilidades como hipertensión (50-60\%), obesidad (15-20\%), diabetes (30-40\%), enfermedades cardiovasculares (12-25\%) y enfermedades respiratorias $(20-25 \%)$.

El hallazgo macroscópico más frecuentemente descrito fue la presencia de pulmones pesados y edematizados además de superficies con áreas azulrojizas, con descripción en una publicación de cambios enfisematosos en todos los pulmones con adherencias pleurales en 7 pacientes. Además, se describió presencia de material trombótico en ramas de las arterias pulmonares en 11 pacientes, esta se asoció con hemorragia parenquimatosa pulmonar e infarto hemorrágico de pulmón con bronconeumonía focal en 8 casos (9).

A nivel de tráquea, bronquios y bronquiolos, se encontraron tráqueas levemente eritematosas, traqueo-bronquitis, en algunos casos espuma rosada en la disección de los bronquios, de igual manera se encontró una secreción mucosa blanca y espesa (10)(4)(11). Fue común encontrar trombos y émbolos, que obstruían las principales arterias pulmonares en 4 casos en la publicación de Bryce y colaboradores (11). Además, se describen trombos dentro de pequeños vasos periféricos (12) y, en la publicación de Lax y colaboradores (9), se encontró material trombótico en las ramas de las arterias pulmonares en todos los casos, lo cual estuvo asociado con hemorragia parenquimatosa pulmonar.

En cuanto a los hallazgos microscópicos se evidenciaron distintos patrones de lesión pulmonar aguda, siendo la más característica el daño alveolar difuso (DAD) que se presentó en el $88 \%$ de los casos, acompañado por formación de membranas hialinas, neumocitos tipo II hiperplásicos con citomegalia, núcleos eosinófilos, nucleolos prominentes, multinucleación, metaplasia escamosa y macrófagos proliferantes y cargados de hemosiderina (10)(13)(14)(8). En la publicación de Lax y colaboradores (9) se identificaron tres etapas del DAD, la primera, precoz con edema y membranas hialinas que corresponde a la fase aguda, una segunda etapa con proliferación de neumocitos hiperplásicos tipo II acompañados de linfocitos y neutrófilos organizando una membrana hialina residual y una tercera etapa tardía con proliferación de fibroblastos, concluyendo principalmente que a mayor duración de la enfermedad hubo mayor grado de organización. También fue frecuente encontrar estas fases de manera superpuesta en distintas áreas pulmonares, por lo que en un mismo pulmón podían encontrarse distintos patrones (15)(16)(14). Otros hallazgos que vale la pena mencionar fueron la aparición de edema intersticial y distintos grados de infiltración linfocítica, así como de macrófagos o infiltración neutrofílica en un 32\% (16)(9).

Se encontraron cambios estructurales en los capilares que constituyeron tres características histológicas: Lesión endotelial, alteración de las membranas de las células endoteliales, y la angiogénesis intususceptiva, en la cual, el alto grado de endotelialitis y trombosis pulmonar llevó a la 
formación de un nuevo vaso sanguíneo a partir de otro preexistente (17). En cuanto a esta última, Grosse y colaboradores (12) identificaron cinco tipos de oclusiones vasculares: microtrombos en pequeñas arteriolas del tabique alveolar, trombos fibrinosos no organizados en arterias pulmonares de calibre medio, trombos parcialmente organizados en arterias pulmonares de calibre medio, émbolos de medula ósea y tromboembolia pulmonar séptica.

Los tipos más comunes de oclusión vascular trombótica/tromboembólica fueron los microtrombos capilares (6), algunos de ellos formados por depósitos de fibrina y otros en conjunto con componentes como plaquetas y colágeno, este hallazgo se describe en el $46 \%$ de los casos analizados (12). En cuanto a la presencia de estos se concluyó que son el resultado de la rección entre los mecanismos reparadores por el daño alveolar y endotelial del DAD que en casos tardíos afecta la proporción de fibrinólisis y coagulación, lo que explica las coagulopatías encontradas en algunos pacientes con COVID-19 (6). En algunos casos se encontraron células gigantes y megacariocitos, como en la publicación de Edler y colaboradores (18), quienes reportaron cuatro casos en los que predominó el cuadro de bronconeumonía focal confluente dominada por granulocitos, acompañada de bronquitis crónica y exacerbada; también se presentaron formas mixtas de DAD y neumonía purulenta en diferentes etapas de organización (18).

Otro patrón de lesión pulmonar que está relacionado con el DAD es la neumonía fibrinosa y organizada aguda (AFOP, del inglés: acute fibrinous and organizing pneumonia), que se diferencia del DAD en que la fibrina intraalveolar organizada constituye el hallazgo histológico dominante. Dentro de los conductos alveolares se encuentran bolas de fibrina, las cuales diversos autores consideran que pueden reflejar la vía común final luego de la fase exudativa, por lo que puede también encontrarse ese patrón en una misma autopsia (19). Esta relación ya había sido comentada antes por Copin M y colaboradores, quienes también se comentan los distintos fenotipos pulmonares, tipo L caracterizado por baja elastancia y bajo peso molecular, y tipo $\mathrm{H}$ caracterizado por alta elastancia y peso pulmonar elevado, en este estudio, cinco de las seis autopsias analizadas presentaron fenotipo tipo $\mathrm{H}$ y AFOP, concluyendo que en la infección grave por SARS-CoV-2 se desplaza el DAD para ser característica la AFOP (20).

En hallazgos encontrados por inmunotinción, según el estudio de Martines y colaboradores se detectó SARS-CoV-2 en las vías respiratorias superiores en el $50 \%$ de los pacientes y en los pulmones en el $92 \%$ de ellos (8). Además, se observaron antígenos virales en las vías respiratorias superiores, epitelio bronquiolar, epitelio de glándulas submucosas, neumocitos tipo I y tipo II, macrófagos alveolares y en membranas hialinas en el pulmón (8).

Por su parte, el examen de microscopía electrónica de los tejidos respiratorios mostró viriones con prominentes proyecciones superficiales característicos de la familia Coronaviridae. En el pulmón, estos viriones se encontraban libres en el espacio alveolar. En las vías respiratorias superiores, se observaron extracelularmente entre los cilios y dentro del citoplasma de las células epiteliales respiratorias. También se detectaron intracelularmente en neumocitos tipo II, y en fagosomas de macrófagos alveolares. Asimismo, se hallaron partículas virales asociadas con fibrina o con las membranas hialinas dentro de los espacios alveolares (8).

Además, se han encontrado infiltrados de neutrófilos y sus trampas extracelulares en asociación con células CD4+ que se encontraron mezcladas con células $\mathrm{CD} 8+$ en los espacios intersticiales y rodeando los bronquiolos más grandes (10)(12)(13).

\section{DISCUSIÓN}

A partir de los artículos revisados sobre los estudios histopatológicos del tejido pulmonar en pacientes con COVID-19, se encontró que los principales hallazgos son el DAD e inflamación en las vías respiratorias $(15)(4)$.

Varios autores han descrito las lesiones histológicas en pacientes fallecidos por COVID-19, las cuales 
incluyen patrones epiteliales, vasculares y fibróticos de lesión pulmonar (60). En primera instancia, los especímenes de tejido pulmonar mostraron DAD con exudados celulares fibromixoides, descamación de neumocitos y membranas hialinas, acompañados de los principales hallazgos patológicos del SDRA en fase temprana. Este último comienza con la lesión de los neumocitos tipo II generando una activación endotelial que culmina en un daño al endotelio de los capilares pulmonares presentando "fugas" y la formación de un edema intersticial e intraalveolar, el daño y la necrosis de los neumocitos tipo II produce entonces anomalías en el surfactante, comprometiendo aún más el intercambio gaseoso alveolar. La presencia de membranas hialinas en los hallazgos histológicos se debe a que el líquido del edema, rico en proteínas, y detritos procedentes de las células epiteliales alveolares muertas, se organiza de esa manera. Además, también están presentes, infiltrados inflamatorios mononucleares intersticiales (principalmente linfocíticos) (6).

Se realizó la búsqueda de partículas virales y se encontró la presencia de viriones raros en el citoplasma de los neumocitos y en el epitelio de las vías respiratorias conductoras (tráquea, bronquios y bronquiolos), además de macrófagos alveolares, sugiriendo que la infección de estos tipos celulares puede ser clave en la replicación y el tráfico viral (15). Los antígenos del SARS-CoV-2 fueron detectados por inmunohistoquímica en células epiteliales ciliadas en la mayoría de los casos y hasta dieciséis días después del inicio de los síntomas conocidos. Esto limita con lo esperado ya que las células del epitelio respiratorio son uno de los primeros tipos celulares con los que se encuentra el virus inhalado.

Sumado a lo anterior, el análisis ultraestructural demostró numerosas partículas virales extracelulares a lo largo de la superficie ciliada y dentro de las células epiteliales columnares ciliadas. Estos hallazgos corroboran los informes de altas cargas virales en el tracto respiratorio superior y sustenta la idea de que las personas infectadas con SARS-CoV2 transmitan fácilmente el virus, con diseminación viral prolongada $\mathrm{y}$ continua en casos graves.
Además, esto puede sugerir que el virus permanece en el tejido pulmonar durante un tiempo y puede ser este el posible mecanismo que conduce y alimenta el daño pulmonar (61).

Otros hallazgos histopatológicos de suma importancia que fueron encontrados en los pulmones son la congestión capilar y los microtrombos. En un estudio se destacó la presencia de exudación moderada de fibrina intraalveolar correspondiente a DAD exudativo, y bronconeumonía superpuesta, que se consideró, tanto en un estado agudo como en un estado organizativo, ser el resultado de una infección bacteriana y no un resultado directo del daño del tejido pulmonar inducido por el SARSCoV-2. Y aunque la presencia de microtrombos puede ser una característica del DAD, esto puede ser más bien una correlación histológica de las coagulopatías descritas en pacientes con COVID-19 (4). Varios estudios informaron otras lesiones además de los aspectos morfológicos indicativos de SDRA, en particular una descripción más consistente de lesión microvascular. Algunos autores describieron capilaritis neutrofílica grave en tres autopsias por COVID-19 (11).

Además, se informó de una relación entre las trampas extracelulares neutrofílicas aberrantes y la presencia de daño orgánico tanto en el parénquima alveolar como en las vías respiratorias. La lesión vascular también se detectó sistemáticamente en algunos pacientes que fallecieron en diferentes etapas de la enfermedad, mostrando neumonía linfocítica y AFOP (60). De la misma manera, se han hecho investigaciones sobre alteraciones estructurales de los capilares y su relación con la angiogénesis intususceptiva presentada dentro de los mismos, donde se encontró que el grado de esta aumentaba significativamente con el aumento de la duración de la hospitalización y se planteó que el mayor grado de endotelialitis y trombosis en los pulmones de los pacientes con COVID-19 puede contribuir a la frecuencia relativa de brotes y angiogénesis intususceptiva observada, la cual es el mecanismo angiogénico predominante incluso en las últimas etapas de la lesión pulmonar crónica (17). 
Tabla 1. Resumen de hallazgos macroscópicas y microscópicos a nivel de sistema respiratorio en pacientes con COVID-19

\begin{tabular}{|c|c|c|c|}
\hline & $\begin{array}{c}\text { Número de casos } \\
\text { Edad (media) } \\
\text { Sexo }\end{array}$ & $\begin{array}{l}\text { Principales hallazgos } \\
\text { macroscópicos }\end{array}$ & Principales hallazgos microscópicos \\
\hline $\begin{array}{l}\text { Martines RB } \\
\text { et al. }(8)\end{array}$ & $\begin{array}{l}8 \\
73,5 \text { años } \\
4 \mathrm{H} / 4 \mathrm{M}\end{array}$ & $\begin{array}{l}\text { Pulmones pesados, edematizados, en } \\
\text { algunos casos se presentó inflamación } \\
\text { en la tráquea y bronconeumonías } \\
\text { focales. }\end{array}$ & $\begin{array}{l}\text { DAD en vías respiratorias superiores e inferiores, } \\
\text { neumocitos hiperplásicos, membranas hialinas, } \\
\text { edema alveolar y depósitos de fibrina. Macrófagos } \\
\text { alveolares hiperplásicos y cargados de } \\
\text { hemosiderina. Metaplasia } \\
\text { Bronconeumonía difusa. }\end{array}$ \\
\hline
\end{tabular}

\begin{tabular}{lll}
\hline $\begin{array}{l}\text { Wichmann et } \\
\text { al.(14) }\end{array}$ & $\begin{array}{l}\text { P3 años, } \\
9 \mathrm{H} / 3 \mathrm{M}\end{array}$ & $\begin{array}{l}\text { Pulmones congestionados y pesados en } \\
10 / 12 \text { pacientes, superficies con } \\
\text { pleuresía leve y áreas pálidas con azul } \\
\text { rojizo profundo, embolia pulmonar. }\end{array}$ \\
\hline $\begin{array}{l}\text { Barton L et } \\
\text { al.(21) }\end{array}$ & 2 59,2 años & $\begin{array}{l}\text { Peso elevado, ambos pulmones firmes, } \\
\text { edematizados difusamente, un caso con } \\
\text { adherencias pleurales. Vías respiratorias } \\
\text { permeables con una mucosa lisa, } \\
\text { brillante y de color crema pálido. }\end{array}$ \\
\hline
\end{tabular}

\begin{tabular}{ll}
\hline Menter Tet & 21 \\
al.(4) & 76 años \\
& $17 \mathrm{H} / 4 \mathrm{M}$
\end{tabular}

Pulmones pesados, firmes, de color

$17 \mathrm{H} / 4 \mathrm{M} \quad 1 / 3$ presento traqueo bronquitis grave. $1 / 3$ presento traqueo bronquitis grave. Enfisema, embolia y hemorragia pulmonar.

DAD, membranas hialinas, neumocitos II hiperplásicos, tromboembolia microvascular, congestión capilar y edema intersticial. Infiltración inflamatoria. Metaplasia escamosa.

DAD agudo, membranas hialinas, inflamación compuesta principalmente por linfocitos, trombos en pequeñas ramas de la arteria pulmonar, inflamación crónica leve dentro de los bronquios y bronquiolos con edema en la mucosa.

16 pacientes presentaron DAD exudativo y 8 pacientes presentaron DAD proliferativo. Membranas hialinas con neumocitos reactivos y células sincitiales. Congestión capilar severa y microtrombos.

\begin{tabular}{lll}
\hline Carsana L et & 38 & Pulmones pesados, congestionados, \\
al.(15) & 69 años & edematosos.
\end{tabular}

DAD; fases exudativas y proliferativas temprana/intermedia superpuestas, trombos plaquetario-fibrina en pequeños vasos arteriales. Neumocitos II hiperplásicos, proliferación de miofibroblastos y fibrosis.

\begin{tabular}{lll}
\hline Ackermann & 7 & Pulmones pesados y edematizados. \\
Met al..(17) & 74 años & \\
& $5 \mathrm{H} / 2 \mathrm{M}$ &
\end{tabular}

DAD con necrosis de las células de revestimiento alveolar, neumocitos II hiperplásicos, fibrina intraalveolar, edema intersticial y linfocitos en el epitelio de los espacios aéreos. Trombos en arterias pulmonares y venas. Capilares deformados y angiogénesis intususceptiva.

\begin{tabular}{lll}
\hline Bradley B et & 14 & Pulmones pesados, edematosos con \\
al..(16) & 73.5 años & presencia de microtrombos pulmonares \\
& (?) H / M & en cinco pacientes. 2 pacientes \\
& & presentaron microtrombos en la tráquea.
\end{tabular}

DAD agudo o agudo y organizado. Fibrina intraalveolar, membranas hialinas o tejido conectivo en paredes del tabique alveolar. Hemorragia, inflamación, infiltración neutrofílica. Submucosa traqueal con edema. Microtrombos pulmonares y en la tráquea.

\begin{tabular}{lll}
\hline Edler Cet & 57 casos de 80 & Peso pulmonar medio combinado de \\
al.(18) & 79,2 años & $1610 \mathrm{~g}$, algunas superficies pulmonares \\
& (?) $\mathrm{H} / \mathrm{M}$ & con signos de pleuresía.
\end{tabular}

DAD con neumocitos tipo II activados, fibroblastos y membranas hialinas, metaplasia escamosa y fibrosis. Células gigantes y megacariocitos. Bronconeumonía focal con bronquitis. Formas mixtas de DAD.

\begin{tabular}{lll}
\hline $\begin{array}{l}\text { Bryce Cet } \\
\text { al.(11) }\end{array}$ & 25 casos de 67 & Peso pulmonar elevado con apariencia \\
& 69 años & irregular, se presentaron émbolos \\
& (?) H / M & $\begin{array}{l}\text { pulmonares que obstruían las } \\
\text { principales arterias pulmonares en } 4 \\
\text { casos. }\end{array}$
\end{tabular}

\begin{tabular}{lll}
\hline Cipolloni $L$ & 2 & Pulmones pesados, espuma rosada en \\
et al. (10) & 56 años & $\begin{array}{l}\text { las vías respiratorias en la disección de } \\
\text { los bronquios, parénquima difusamente }\end{array}$ \\
\hline
\end{tabular}

DAD en fase aguda o proliferativa temprana en 22 casos, neumocitos tipo II hiperplásicos y células multinucleadas. Inflamación linfocítica intersticial leve y difusa. Áreas de proliferación capilar con inflamación y lesión similar a capilaritis en 14 casos. Trombos de fibrina intravascular.

Caso 1 neumonía organizada grave, células infiltrantes plasmáticas y macrófagos, edema y septos engrosados. Fibrina en vasos y paredes. 
edematoso y firme. Pequeñas áreas fibróticas blanquecinas.

$\begin{array}{lll}\text { Grosse Cet } & 14 & \text { Pulmones pesados, firmes y } \\ \text { al.(13) } & 82 \text { años } & \text { edematizados con congestión vascular } \\ & 9 \mathrm{H} / 5 \mathrm{M} & \begin{array}{l}\text { bilateral masiva y parcheado } \\ \text { homogéneo de rojo oscuro a grisáceo, } \\ \text { derrames pleurales serosos leves y } \\ \text { paciente pleuritis fibrinosa leve. }\end{array}\end{array}$

\begin{tabular}{ll}
\hline Lax $S$ et & 11 \\
al.(9) & 66 años
\end{tabular}

$8 \mathrm{H} / 3 \mathrm{M}$

$\begin{array}{ll}\text { Fox } S \text { et } & 4 \\ \text { al.(12) } & 60 \text { años }\end{array}$

(?) $\mathrm{H} / \mathrm{M}$
Pulmones pesados, congestión bilateral masiva, superficies azul-rojizas, cambios enfisematosos, sistema bronquial con moco viscoso. Trombos en ramas de arterias pulmonares, hemorragia. Infarto de pulmón con bronconeumonía focal en la mitad de los casos.

Pulmones pesados, edematizados y superficies hemorrágicas. Bronquios con mucosa blanca y espuma rosada en vías respiratorias. Tráqueas eritematosas. Trombos dentro de vasos periféricos.

Pulmones congestionados, edematizados con áreas difusas y de consolidación. Inflamación de vías respiratorias en 38 casos.
Microtrombos y angiogénesis intususceptiva. Caso $2 \mathrm{DAD}$ en fase proliferativa, infiltración de fibroblastos, macrófagos con neumocitos II hiperplásicos. Microtrombos.

DAD; fase organizativa/proliferativa con proliferación fibroblástica, neumocitos II hiperplásicos, metaplasia escamosa y macrófagos. DAD en fase aguda, membranas hialinas, congestión capilar, edema intersticial, inflamación linfocítica y descamación del epitelio alveolar. DAD en fase aguda y crónica. Microtrombos y trombos.

DAD; tres etapas, la primera con edema y membranas hialinas, segunda etapa con proliferación de neumocitos II hiperplásicos, linfocitos y neutrófilos organizando una membrana hialina residual y una tercera etapa con proliferación de fibroblastos. Microtrombos y trombos de arterias pequeñas.

DAD bilateral, infiltrado linfocitico alrededor de bronquiolos y pequeños vasos que contenían trombos. Neumocitos II hiperplásicos en espacios alveolares, presencia de membranas hialinas y depósito de fibrina. Capilares alveolares engrosados. Infiltrado neutrofílico y trampas extracelulares.

Dos patrones; DAD con membranas hialinas, neumocitos II hiperplásicos y neumonía organizada con metaplasia escamosa en mayor duración de la enfermedad. 44 pacientes con microtrombos y lesión vascular en 11.

Pulmones aumentados, consolidados, DAD en dos fases; fase exudativa con membranas superficie ligeramente pegajosa de color hialinas prominentes y fase organizativa con rojo oscuro y presencia de derrames descamación, metaplasia escamosa de células pleurales. epiteliales, membranas hialinas organizadas e infiltración de células inflamatorias en tabiques alveolares. Neumocitos II hiperplásicos. Hemorragia intraalveolar y congestión vascular.

\begin{tabular}{lll}
\hline $\begin{array}{l}\text { Magro C et } \\
\text { al.(24) }\end{array}$ & $\begin{array}{l}2 \text { casos de } 5 \\
65 \text { años } \\
2 \mathrm{H} .\end{array}$ & $\begin{array}{l}\text { Pulmones } \\
\text { hemorrágicos. }\end{array}$ \\
\hline $\begin{array}{l}\text { Suess Cet } \\
\text { al.(25) }\end{array}$ & $\begin{array}{l}\text { 59 años } \\
\mathrm{H}\end{array}$ & $\begin{array}{l}\text { Pulmones agrandados, edematizados y } \\
\text { parénquima gomoso con áreas } \\
\text { hemorrágicas. Bronquios llenos de } \\
\text { líquido. }\end{array}$
\end{tabular}

DAD con neumonitis hemorrágica organizada con depósito de fibrina dentro de vasos con necrosis de células endoteliales.

DAD en estadio temprano con marcada formación de membrana hialina. neumocitos II hiperplásicos, exudados proteínicos, hemorragia alveolar y depósitos de fibrina intraalveolar. Microtrombos en capilares y trombos en arterias pulmonares. Metaplasia bronquial con fibrosis circundante del intersticio.

\begin{tabular}{lll}
\hline Falasca L et & 22 & Pulmones aumentados, firmes, \\
al.(26) & 62 años & edematosos y congestionados con \\
& $15 \mathrm{H} / 7 \mathrm{M}$ & engrosamiento y derrame pleural.
\end{tabular}
DAD exudativa y proliferativa, membranas hialinas. neumonía organizada con fibrosis y neumocitos II hiperplásicos. Lesión vascular con trombos y vasculitis. Partículas de SARS-CoV-2 dentro de los neumocitos de tipo II

Del Nonno $F \quad 1 \quad$ Pulmones congestionados, edematosos DAD en distintos estadios, con edema y fibrosis et al.(27) 61 años y hemorrágicos de color oscuro. Pleura intersticial. Capilares engrosados, extravasación 


\begin{tabular}{|c|c|c|}
\hline & $\mathrm{M}$ & $\begin{array}{l}\text { con adherencias fibrosas. Oclusión } \\
\text { debido a trombo en la arteria pulmonar } \\
\text { principal. }\end{array}$ \\
\hline $\begin{array}{l}\text { Stoyanov } G \\
\text { et al. }(28)\end{array}$ & $\begin{array}{l}2 \\
55 \text { años } \\
1 \mathrm{H} / 1 \mathrm{M}\end{array}$ & $\begin{array}{l}\text { Pulmones agrandados, cianóticos, } \\
\text { contraídos hacia el hilo y en } \\
\text { consolidación difusa. Tráqueas y } \\
\text { arboles bronquiales edematosos con } \\
\text { eritema severo en áreas intercondrales } \\
\text { de la mucosa. }\end{array}$ \\
\hline $\begin{array}{l}\text { Bosmuller } H \\
\text { et al.(29) }\end{array}$ & $\begin{array}{l}4 \\
71 \text { años } \\
3 \mathrm{H} / 1 \mathrm{M}\end{array}$ & $\begin{array}{l}\text { Pulmones aumentados, edematizados y } \\
\text { consolidación difusa. } 2 \text { casos con } \\
\text { presencia de trombos en vasos } \\
\text { pulmonares. }\end{array}$ \\
\hline $\begin{array}{l}\text { Scendoni } R \\
\text { et al. }(30)\end{array}$ & $\begin{array}{l}2 \\
53 \text { años } \\
1 \mathrm{H} / 1 \mathrm{M}\end{array}$ & $\begin{array}{l}\text { Pulmones } \\
\text { congestionados. }\end{array}$ \\
\hline $\begin{array}{l}\text { Xiu-Wu et } \\
\text { al.(31) }\end{array}$ & $\begin{array}{l}37 \\
\text { (?) años } \\
\text { (?) H y (?)M }\end{array}$ & $\begin{array}{l}\text { Cambios en el parénquima pulmonar, } \\
\text { tráquea y bronquios congestionados, } \\
\text { aumento de la secreción y exfoliación } \\
\text { epitelial. }\end{array}$ \\
\hline $\begin{array}{l}\text { Aguiar D et } \\
\text { al.(32) }\end{array}$ & $\begin{array}{l}1 \\
31 \text { años } \\
1 \mathrm{M}\end{array}$ & $\begin{array}{l}\text { Pulmones pesados, firmes, gomosos y } \\
\text { edematizados con áreas de } \\
\text { consolidación blanquecina. Derrame } \\
\text { traqueobronquial y pleural. }\end{array}$ \\
\hline
\end{tabular}

\begin{tabular}{ll}
\hline Konopka K & 1 \\
et al.(33) & 37 años \\
& $1 \mathrm{H}$
\end{tabular}

\begin{tabular}{ll}
\hline ChmielikE & 3 \\
et al.(34) & 61 años \\
& $3 \mathrm{H}$
\end{tabular}

\begin{tabular}{lll}
\hline $\begin{array}{l}\text { Ducloyer } M \\
\text { et al.(35) }\end{array}$ & 1 & Pulmones ligeramente densos. \\
& $1 \mathrm{H}$ &
\end{tabular}

\begin{tabular}{lll}
\hline Remmelink & 17 & Pulmones pesados y parénquima \\
Met al.(36) & 72 años & pulmonar con área de hemorragia rojo. \\
& $12 \mathrm{H} / 5 \mathrm{M}$ & $\begin{array}{l}\text { Trombos en grandes arterias } \\
\text { pulmonares en 2 pacientes y } \\
\text { adherencias pleurales en 4. }\end{array}$ \\
\end{tabular}

\begin{tabular}{ll}
\hline Maximilian & 3 \\
Let al.(37) & 48 años \\
& $3 \mathrm{H}$
\end{tabular}

Pulmones pesados con taponamiento de moco en vías respiratorias conductoras y consolidación del parénquima pulmonar. pleuras sin adherencias.

Pulmones congestionados,
tromboembolias pulmonares bilaterales y áreas hemorrágicas en un caso. de eritrocitos a espacios alveolares y trombos vasculares.

DAD con membranas hialinas alveolares, neumonía intersticial, microtrombos y áreas de proliferación fibroblástica. Neumocitos II hiperplásicos. Descamación severa del epitelio respiratorio bronquial. Cambios degenerativos y necróticos en arterias.

3 casos presentaron DAD, membranas hialinas, macrófagos intraalveolares con hiperplasia $\mathrm{y}$ descamación del epitelio, uno de ellos en etapa organizativa con exudados fibrinosos extensos y engrosamiento de los tabiques alveolares.

y Un caso con neumonía intersticial aguda en fase exudativa, un segundo caso con fase proliferativa de DAD con membranas hialinas y moderada organización fibrótica.

DAD e inflamación exudativa con fibrina en espacios alveolares, membranas hialinas, neumocitos II hiperplásicos, acumulación de mucina en ciertas áreas. Vasos pulmonares con vasculitis, trombosis y tromboembolismo.

DAD en fase exudativa con membranas hialinas, depósito de fibrina y neumocitos II hiperplásicos. Aumento de macrófagos intraalveolares, neutrófilos y linfocitos. Bronquios y bronquiolos con infiltración linfocítica en sus paredes.

DAD con neumocitos II hiperplásicos, engrosamiento intersticial, edema, membranas hialinas dispersas, trombos de fibrina dentro de vasos.

Pulmones firmes en 2 pacientes y 1 paciente con DAD, membranas hialinas, neumocitos hiperplásicos y macrófagos alveolares. 1 paciente con bronconeumonía. 2 casos con microtrombos y el otro paciente con tromboembolismo.

DAD en una fase aguda con membranas hialinas, edema alveolar, exudado de eosinófilos y macrófagos vacuolados, y una fase organizada con agrandamiento de tabiques alveolares, depósito de fibrina alveolar, neumocitos II hiperplásicos.

DAD con edema intersticial e intraalveolar, membranas hialinas, deposición de fibrina, infiltrado inflamatorio mononuclear y neumocitos II hiperplásicos. Microtrombos en pequeñas arterias pulmonares en 11 pacientes. Pulmón derecho colapsado, superficies marrones, líquido en cavidad pleural izquierda y árbol traqueobronquial revestido por mucosa hiperémica en otro caso.
DAD con membranas hialinas, exudado fibrinoso, inflamación focal, neumocitos II hiperplásicos. Metaplasia escamosa y hemorragia intraalveolar. Microtrombos en arterias pulmonares en un caso, este mismo con depósitos de fibrina en alveolos. 


\begin{tabular}{|c|c|c|c|}
\hline $\begin{array}{l}\text { Sauter Jet } \\
\text { al.(38) }\end{array}$ & $\begin{array}{l}8 \\
57.5 \text { años } \\
4 \mathrm{H} / 4 \mathrm{M}\end{array}$ & $\begin{array}{l}\text { Pulmones pesados, bronquitis y } \\
\text { bronquiolitis en todos los casos. }\end{array}$ & $\begin{array}{l}\text { DAD en fase aguda con membranas hialinas, } \\
\text { edema alveolar e intersticial, neumocitos II en } \\
\text { proliferación, fibrina alveolar y descamación de } \\
\text { neumocitos en espacios alveolares. DAD en fase } \\
\text { organizada con engrosamiento de pared alveolar, } \\
\text { proliferación fibroblástica y de neumocitos II, } \\
\text { edema pulmonar. }\end{array}$ \\
\hline $\begin{array}{l}\text { Calvin Met } \\
\text { al.(39) }\end{array}$ & $\begin{array}{l}1 \\
19 \text { años } \\
1 \mathrm{H}\end{array}$ & $\begin{array}{l}\text { Pulmones edematosos, firmes con } \\
\text { alternancia de zonas pálidas y rojas. }\end{array}$ & $\begin{array}{l}\text { DAD con edema, membrana hialina, pequeños } \\
\text { vasos sanguíneos con trombos de fibrina. }\end{array}$ \\
\hline $\begin{array}{l}\text { Tachikawa } \\
\text { Net al.(40) }\end{array}$ & $\begin{array}{l}1 \\
93 \text { años } \\
1 \mathrm{M}\end{array}$ & $\begin{array}{l}\text { Pulmones edematosos, pesados, } \\
\text { superficies firmes, color rojo pardusco y } \\
\text { exudado viscoso. }\end{array}$ & $\begin{array}{l}\text { DAD en distintas fases, neumocitos degenerados, } \\
\text { membranas hialinas, hemorragia alveolar focal. } \\
\text { Tráquea, bronquios y bronquiolos presentan } \\
\text { inflamación aguda en forma focal células } \\
\text { epiteliales descamadas e infiltración neutrofílica. } \\
\text { Bronconeumonía aguda. Trombos en arterias } \\
\text { pulmonares. }\end{array}$ \\
\hline
\end{tabular}

\begin{tabular}{lll}
\hline Zeng Z et & 1 & No descrito. \\
al.(41) & 55 años & \\
& $1 \mathrm{M}$ &
\end{tabular}

Inflamación exudativa, áreas de exudación serosa con edema pulmonar, neumocitos II hiperplásicos, septos alveolares ensanchados, hiperemia y dilatación de capilares. Células inflamatorias alrededor de pequeños vasos sanguíneos. Dilatación y congestión capilar alrededor de un nódulo pulmonar.

\begin{tabular}{lll}
\hline $\begin{array}{l}\text { Flikweert } A \\
\text { et al.(42) }\end{array}$ & 7 & No descrito. \\
& $\begin{array}{l}73 \text { años } \\
5 \text { H y } 2 \mathrm{M}\end{array}$ \\
& \\
\hline $\begin{array}{l}\text { Zhang H et } \\
\text { al.(43) }\end{array}$ & $\begin{array}{l}72 \text { años } \\
1 \mathrm{H}\end{array}$ & No descrito. \\
& 7 & \\
\hline $\begin{array}{l}\text { Taghi M et } \\
\text { al.(44) }\end{array}$ & 67,85 años & No descrito. \\
& $5 \mathrm{H} / 2 \mathrm{M}$ & \\
\hline
\end{tabular}

DAD, neumonía organizada, fibrosis, neumocitos II hiperplásicos, células gigante multinucleadas, microtrombos y ensanchamiento de septos alveolares.

DAD en fase organizativa con neumocitos II hiperplásicos, células de revestimiento alveolar desnudas y exudados fibrinosos intraalveolares.

DAD en fase aguda con membranas hialinas, edema en la pared alveolar, exudado fibrinoide. $\mathrm{DAD}$ en fase proliferativa y fibrótica. Edema e inflamación en espacios alveolares presente en todos los casos.

\begin{tabular}{|c|c|c|c|}
\hline $\begin{array}{l}\text { Xiaohong } Y \\
\text { et al.(45) }\end{array}$ & $\begin{array}{l}3 \\
70 \text { años } \\
2 \mathrm{H} / 1 \mathrm{M}\end{array}$ & No descrito. & $\begin{array}{l}\text { Exudación serosa en cavidad alveolar, membranas } \\
\text { hialinas, capilares dilatados y congestionados, } \\
\text { paredes alveolares engrosados, trombosis hialina } \\
\text { en pequeños vasos, neumocitos II hiperplásicos. }\end{array}$ \\
\hline $\begin{array}{l}\text { Recalde B et } \\
\text { al.(46) }\end{array}$ & $\begin{array}{l}10 \\
78.6 \text { años } \\
5 \mathrm{H} / 5 \mathrm{M}\end{array}$ & No descrito. & $\begin{array}{l}\text { DAD en distintos estadios, membranas hialinas, } \\
\text { neumocitos II hiperplásicos, inflamación crónica } \\
\text { leve. Trombos de vasos de tamaño mediado e } \\
\text { hiperplasia del musculo liso vascular. }\end{array}$ \\
\hline $\begin{array}{l}\text { Xu Z et } \\
\text { al.(47) }\end{array}$ & $\begin{array}{l}1 \\
50 \text { años } \\
1 \mathrm{H}\end{array}$ & No descrito. & $\begin{array}{l}\text { DAD con exudados fibromixoides celulares, } \\
\text { descamación de neumocitos, membranas hialinas, } \\
\text { infiltrados mononucleares, }\end{array}$ \\
\hline $\begin{array}{l}\text { Ding M et } \\
\text { al.(48) }\end{array}$ & $\begin{array}{l}32 \\
63,2 \text { años } \\
13 \mathrm{H} / 19 \mathrm{M}\end{array}$ & No descrito. & $\begin{array}{l}\text { Fibrosis pulmonar, edema alveolar intersticial, } \\
\text { hiperplasia epitelial, y formación de nódulos } \\
\text { granulomatosos. }\end{array}$ \\
\hline $\begin{array}{l}\text { Beth M et } \\
\text { al.(49) }\end{array}$ & $\begin{array}{l}17 \\
62 \text { años } \\
10 \mathrm{H} / 7 \mathrm{M}\end{array}$ & No descrito. & $\begin{array}{l}\text { DAD y AFOP con bolas de fibrina en espacios } \\
\text { alveolares y bronquiolos. En todos los casos se } \\
\text { presentó neumocitos II hiperplásicos, neutrófilos, } \\
\text { ensanchamiento intersticial edematoso y trombos } \\
\text { vasculares en dos casos. }\end{array}$ \\
\hline
\end{tabular}




\begin{tabular}{lll}
\hline Valvidia $M$ & 18 & No descrito. \\
et al.(50) & 61 años & \\
& $10 \mathrm{H} / 8 \mathrm{M}$ &
\end{tabular}

\begin{tabular}{lll}
\hline Schaefer I et & 7 & No descrito. \\
al.(51) & 62.4 años & \\
& $5 \mathrm{H} / 2 \mathrm{M}$ &
\end{tabular}

\begin{tabular}{lll}
\hline $\begin{array}{l}\text { Barisione E } \\
\text { et al.(52) }\end{array}$ & 8 & No descrito. \\
& 76 años & \\
& $6 \mathrm{H} / 2 \mathrm{M}$ &
\end{tabular}

\begin{tabular}{lll}
\hline Pernazza $A$ & 1 & No descrito. \\
et al.(53) & 61 años & \\
& $1 \mathrm{H}$ &
\end{tabular}

\begin{tabular}{lll}
\hline $\begin{array}{l}\text { Elsoukkary } S \\
\text { et al.(54) }\end{array}$ & 32 & No descrito. \\
& 68 años & \\
& H 22 / $10 \mathrm{M}$ &
\end{tabular}

\begin{tabular}{lll}
\hline Junhua Wet & 10 & No descrito. \\
al.(55) & 70 años & \\
& $7 \mathrm{H} / 3 \mathrm{M}$ &
\end{tabular}

\begin{tabular}{lll}
\hline Postigo Met & 7 & No descrito. \\
al.(56) & 75 años & \\
& (?) H / (?) M. &
\end{tabular}

\begin{tabular}{lll}
\hline Wang Zet & 3 & No descrito. \\
al.(57) & 78 años & \\
& $1 \mathrm{H} / 2 \mathrm{M}$ &
\end{tabular}

\begin{tabular}{lll}
\hline Eckerman $M$ & 6 & No descrito. \\
et al.(58) & $(?)$ años & \\
& $5 \mathrm{H} / 1 \mathrm{M}$ & \\
\hline
\end{tabular}

DAD en estadio fibrótico y neumonía organizada, entremezclados con las áreas fibróticas, la mayoría de los casos presentaban congestión del tabique alveolar y grados variables de hemorragia intraalveolar, fibrina, edema y descamación de neumocitos. Neumocitos II hiperplásicos, metaplasia escamosa cerca de estructuras bronquiales, edema, áreas fibróticas. Trombos en 6 casos.

DAD con membranas hialinas, 4 casos en fase aguda con focos de organización dispersa. Todos los pacientes presentaron infiltrados inflamatorios difusos con linfocitos intersticiales. 2 pacientes con DAD organizado con obliteración de espacios alveolares y cicatrización extensa. 5 pacientes con coagulopatías (embolia pulmonar y trombos in situ). Secciones traqueobronquiales con metaplasia escamosa y desprendimiento epitelial. Infiltrado linfocitico y edema en pared de vías respiratorias.

DAD fase exudativa en dos pacientes, con denudación de neumocitos y membrana hialina. DAD fase proliferativa en 3 pacientes con exudados fibrinosos intraalveolares y tapones fibroblásticos organizados. DAD fase organizativa en dos pacientes con proliferación progresiva de fibroblastos intersticiales. Neumocitos II hiperplásicos. 1 paciente con metaplasia escamosa y bronquiolizacion alveolar.

Perdida difusa de neumocitos e hiperplasia reactiva, agrupaciones de macrófagos alveolares e infiltrados inflamatorios intersticiales, marginación neutrofílica prominente y difusa en vasos septales.

DAD en 24 pacientes en fase exudativa $y$ proliferativa. Neumonía organizada en 14 pacientes. Todos los casos presentaron neumocitos II hiperplásicos y metaplasia escamosa bronquial.

DAD en fase aguda y organizativa, exudación de fibrina en cavidad alveolar, neumocitos II reactivos y proliferantes, membranas hialinas, infiltración neutrofílica.

Membranas hialinas, hiperplasia y desprendimiento de neumocitos, hiperplasia de macrófagos alveolares, microtrombosis $\mathrm{y}$ hemorragia intraalveolar.

DAD fase aguda con alveolos colapsados, espacios alveolares con exudados proteínicos, tabiques alveolares ensanchados, neumocitos II hiperplásicos. DAD en fase organizativa con exudados fibrinosos, membrana hialina y metaplasia escamosa en distintos espacios alveolares. Degeneración vascular en células epiteliales.

DAD, membranas hialinas, neumonía intersticial linfocítica moderada, tabiques alveolares agrandados, trombos en pequeñas venas 
pulmonares, hipertrofia de la media muscular en vasos precapilares y poscapilares, enfisema centrolobulillar leve en 5 pacientes.

\begin{tabular}{lll}
\hline Komoss F et & 13 & No descrito. \\
al.(59) & 78 años & \\
& $10 \mathrm{H} / 3 \mathrm{M}$. &
\end{tabular}

DAD asociado con microtrombosis de capilares alveolares en 7 pacientes y 9 presentaron hemorragias intraalveolares. Hiperplasia y metaplasia escamosa de neumocitos en fases posteriores junto infiltración intersticial linfocítica y depósitos de fibras de colágeno.

$\mathrm{DAD}=$ Daño alveolar difuso, $\mathrm{AFOP}=$ Neumonía aguda fibrinosa y organizada $\mathrm{H}=$ Hombres, $\mathrm{M}=$ Mujeres

(?) $\mathrm{M} \mathrm{y} \mathrm{H}=$ No se reportó el número de hombres y mujeres incluidos en el estudio

(?) años $=$ Son se reportó la edad de los pacientes incluidos en el estudio.

Es importante resaltar la relación entre la incidencia de las coagulopatías y un mal pronóstico en los pacientes con COVID-19 (62)(63), tanto la coagulación como la inflamación son mecanismos esenciales para la defensa frente a patógenos, y entre más daño cause la respuesta para eliminar al patógeno más se verá asociada a un peor pronóstico (64)(65). Dentro del grupo de coagulopatías se encontraron, coagulopatía asociada al COVID-19 (CAC), coagulopatía intravascular diseminada (CID) y coagulopatía inducida por sepsis (CIS). La patogenia de la CAC no se encuentra totalmente descifrada pero sus mecanismos se pueden estudiar comparándola con otras coagulopatías ya conocidas (66). En los casos estudiados, las tres coagulopatías se asocian a un nivel elevado de Dímero-D, aumento en la degradación de la fibrina y prolongación del tiempo de protrombina, sobre todo la CAC (66)(67).

Los factores mencionados en conjunto con la trombocitopenia pueden justificar la formación de los coágulos de fibrina entre los hallazgos histopatológicos característicos de pacientes con COVID-19 (67), mediante la excesiva activación de plaquetas y de la cascada de coagulación, además, pacientes con edad avanzada y un recuento bajo de plaquetas se asocia con una alta mortalidad en un plazo de tiempo menor a 28 días (66).

En cuanto a las características citopatológicas en muestras de lavado broncoalveolar de pacientes con COVID-19 se encontró un elevado número de células plasmáticas activadas mezcladas con linfocitos T y células B dispersas, sin embargo, no se encontró material hialino o los neumocitos descamados que se habrían esperado con base en los principales cambios histopatológicos reportados en los pulmones de pacientes con COVID-19. Por lo que se llegó a la conclusión de que la infección por SARS-CoV-2 no presenta características citomorfológicas específicas (6). No obstante, en diversas muestras respiratorias, entre ellas el esputo o el lavado broncoalveolar, podemos observar características citomorfológicas de lesión pulmonar aguda y reparación, como el aumento del número de macrófagos, neumocitos tipo II atípicos, células escamosas metaplásicas y células multinucleadas.

A partir de esto, se puede sospechar una etiología viral basada en cambios citoplasmáticos y nucleares en los macrófagos y las células epiteliales; teniendo en cuenta también la presencia de, agrandamiento celular y nuclear, descamación epitelial, citoplasma espumoso, vacuolas citoplasmáticas paranucleares más grandes, aclaramiento nuclear e inclusiones intranucleares (13)(23)(29)(38).

Por último, los fenotipos en los pulmones se pueden explicar gracias a la clasificación de Gattinoni que los categoriza en, un primer fenotipo con baja elastancia pulmonar indicando que la cantidad de gas en el pulmón es cercana a la normal, con pérdida de la regulación de la perfusión y de la vasoconstricción hipóxica; por otro lado tenemos una elastancia pulmonar elevada que estuvo presente en la mayoría de los pacientes debida al aumento del edema, el cual a su vez genera un aumento de la ventilación y la perfusión por la fracción de gasto cardíaco que perfunde los tejidos no aireados (60). 


\section{CONCLUSIONES}

En síntesis, el principal hallazgo histopatológico a nivel respiratorio en pacientes con COVID-19 es el daño alveolar difuso (DAD), seguido de este y a nivel macroscópico se encuentran los pulmones pesados, edematosos o congestionados. La gravedad de estos hallazgos está determinada por la edad del paciente $y$ el padecimiento de diversas comorbilidades/afecciones sobre todo las relacionadas con el sistema respiratorio y cardiovascular que se asocian con una mayor mortalidad en los pacientes, de igual manera estos factores influyen en el desarrollo de un amplio espectro heterogéneo de hallazgos microscópicos y macroscópicos con diferente incidencia en los casos revisados. Conocer este amplio espectro de hallazgos relacionados con el SARS-CoV-2 proporciona diferentes contextos que puede contribuir a la construcción de guías de tratamientos clínicos más eficaces, por ende, se debería continuar con la recopilación de casos de pacientes confirmados con COVID-19 para tener cada día más información respecto a la patología y poder desarrollar nuevas estrategias de tratamiento.

CONTRIBUCIONES DE LOS AUTORES: AM, LM y SP: concepción y diseño del estudio, recolección análisis e interpretación de datos, discusión de resultados y redacción del borrador del artículo, revisión crítica y aprobación de versión final, responsable de la veracidad e integridad del artículo.

CONFLICTOS DE INTERESES: Los autores no declaran conflictos de intereses.

\section{REFERENCIAS}

1. Ye ZW, Yuan S, Yuen KS, Fung SY, Chan CP, Jin DY. Zoonotic origins of human coronaviruses. Int $\mathrm{J}$ Biol Sci. 2020; 16(10): 1686-97.

2. Chan JFW, Kok KH, Zhu Z, Chu H, To KKW, Yuan S, et al. Genomic characterization of the 2019 novel human-pathogenic coronavirus isolated from a patient with atypical pneumonia after visiting Wuhan. Emerg
Microbes Infect. 2020; 9(1): 221-36.

3. Polak SB, Van Gool IC, Cohen D, von der Thüsen JH, van Paassen J. A systematic review of pathological findings in COVID-19: a pathophysiological timeline and possible mechanisms of disease progression. Mod Pathol [Internet]. 2020; 33(11): 2128-38. Disponible en: http://dx.doi.org/10.1038/s41379-020-0603-3

4. Menter T, Haslbauer JD, Nienhold R, Savic S, Hopfer $\mathrm{H}$, Deigendesch N, et al. Postmortem examination of COVID-19 patients reveals diffuse alveolar damage with severe capillary congestion and variegated findings in lungs and other organs suggesting vascular dysfunction. Histopathology. 2020; 77(2): 198-209.

5. Shi Y, Wang G, Cai X peng, Deng J wen, Zheng L, Zhu $\mathrm{H}$ hong, et al. An overview of COVID-19. J Zhejiang Univ Sci B. 2020; 21(5): 343-60.

6. Calabrese F, Pezzuto F, Fortarezza F, Hofman P, Kern I, Panizo A, et al. Pulmonary pathology and COVID19: lessons from autopsy. The experience of European Pulmonary Pathologists. Virchows Arch. 2020; 477(3): 359-72.

7. Woo PCY, Lau SKP, Chu C, Chan K, Tsoi H, Huang $\mathrm{Y}$, et al. Characterization and Complete Genome Sequence of a Novel Coronavirus, Coronavirus HKU1 , from Patients with Pneumonia. 2005; 79(2): 884-95.

8. Martines RB, Ritter JM, Matkovic E, Gary J, Bollweg BC, Bullock H, et al. Pathology and pathogenesis of SARS-CoV-2 associated with fatal coronavirus disease, united states. Emerg Infect Dis. 2020; 26(9): 2005-15.

9. Lax SF, Skok K, Zechner P, Kessler HH, Kaufmann N, Koelblinger C, et al. Pulmonary Arterial Thrombosis in COVID-19 With Fatal Outcome: Results From a Prospective, Single-Center, Clinicopathologic Case Series. Ann Intern Med. 2020; 173(5): 350-61.

10. Cipolloni L, Sessa F, Bertozzi G, Baldari B, Cantatore S, Testi R, et al. Preliminary post-mortem COVID-19 evidence of endothelial injury and factor VIII hyperexpression. Diagnostics. 2020; 10(8): 1-16.

11. Bryce C, Grimes Z, Pujadas E, Ahuja S, Beasley MB, Albrecht R, et al. Pathophysiology of SARS-CoV-2: targeting of endothelial cells renders a complex disease 
with thrombotic microangiopathy and aberrant immune response. The Mount Sinai COVID-19 autopsy experience. 2020

12. Fox SE, Akmatbekov A, Harbert JL, Li G, Brown JQ. *not peer reviewed* Pulmonary and Cardiac Pathology in Covid-19: The First Autopsy Series from New Orleans 1) Department of Pathology, LSU Health Sciences Center, New Orleans 2) Pathology and Laboratory Medicine Service, Southeast Louisiana Veterans. medRxiv [Internet]. 2020; 2020.04.06.20050575. Disponible en: https://doi.org/10.1101/2020.04.06.20050575

13. Grosse C, Grosse A, Salzer HJF, Dünser MW, Motz $\mathrm{R}$, Langer R. Analysis of cardiopulmonary findings in COVID-19 fatalities: High incidence of pulmonary artery thrombi and acute suppurative bronchopneumonia. Cardiovasc Pathol. 2020; 49 (January).

14. Wichmann D, Sperhake JP, Lütgehetmann M, Steurer S, Edler C, Heinemann A, et al. Autopsy Findings and Venous Thromboembolism in Patients With COVID19: A Prospective Cohort Study. Ann Intern Med. 2020; 173(4): 268-77.

15. Nasr A, Rossi RS, Pellegrinelli A, Zerbi P, Rech R. Pulmonary post-mortem findings in a large series of COVID-19 cases from Northern Italy Since December 2019, an outbreak caused by a new coronavirus infection (severe acute respiratory syndrome coronavirus 2 , SARS-CoV-2) disease was reported, starting. 2020; $1-11$.

16. Bradley BT, Maioli H, Johnston R, Chaudhry I, Fink $\mathrm{SL}, \mathrm{Xu} \mathrm{H}$, et al. Histopathology and Ultrastructural Findings of Fatal COVID-19 Infections. medRxiv. 2020.

17. Ackermann M, Verleden SE, Kuehnel M, Haverich A, Welte $\mathrm{T}$, Laenger F, et al. Pulmonary Vascular Endothelialitis, Thrombosis, and Angiogenesis in Covid-19. N Engl J Med. 2020; 383(2): 120-8.

18. Edler C, Schröder AS, Aepfelbacher M, Fitzek A, Heinemann A, Heinrich F, et al. Erratum: Correction to: Dying with SARS-CoV-2 infection-an autopsy study of the first consecutive 80 cases in Hamburg, Germany (International journal of legal medicine (2020) 1344 (1275-1284). Int J Legal Med. 2020;
134(5): 1977.

19. Nicholson AG, Osborn M, Devaraj A, Wells AU. COVID-19 related lung pathology: old patterns in new clothing? Histopathology. 2020; 77(2): 169-72.

20. Copin MC, Parmentier E, Duburcq T, Poissy J, Mathieu D, Caplan M, et al. Time to consider histologic pattern of lung injury to treat critically ill patients with COVID-19 infection. Intensive Care Med [Internet]. 2020; 46(6): 1124-6. Disponible en: https://doi.org/10.1007/s00134-020-06057-8

21. Barton LM, Duval EJ, Stroberg E, Ghosh S, Mukhopadhyay S. COVID-19 Autopsies, Oklahoma, USA. Am J Clin Pathol. 2020; 153(6): 725-33.

22. Borczuk AC, Salvatore SP, Seshan S V., Patel SS, Bussel JB, Mostyka M, et al. COVID-19 pulmonary pathology: a multi-institutional autopsy cohort from Italy and New York City. Mod Pathol [Internet]. 2020; 33(11): 2156-68. Disponible en: http://dx.doi.org/10.1038/s41379-020-00661-1

23. Adachi $\mathrm{T}$, Chong JM, Nakajima N, Sano M, Yamazaki J, Miyamoto I, et al. Clinicopathologic and immunohistochemical findings from autopsy of patient with COVID-19, Japan. Emerg Infect Dis. 2020; 26(9): 2157-61.

24. Magro C, Mulvey JJ, Berlin D, Nuovo G, Salvatore S, Harp J, et al. Since January 2020 Elsevier has created a COVID-19 resource centre with free information in English and Mandarin on the novel coronavirus COVID- 19. The COVID-19 resource centre is hosted on Elsevier Connect, the company' $\mathrm{s}$ public news and information. 2020; (January).

25. Suess C, Hausmann R. Gross and histopathological pulmonary findings in a COVID-19 associated death during self-isolation. Int J Legal Med. 2020; 134(4): 1285-90.

26. Falasca L, Nardacci R, Colombo D, Lalle E, Di Caro A, Nicastri E, et al. Postmortem Findings in Italian Patients With COVID-19: A Descriptive Full Autopsy Study of Cases With and Without Comorbidities. J Infect Dis. 2020; 222(11): 1807-15.

27. Del Nonno F, Colombo D, Nardacci R, Falasca L. Fatal pulmonary arterial thrombosis in a COVID-19 
patient, with asymptomatic history, occurred after swab negativization. Thromb J. 2021; 19(1): 1.

28. Stoyanov GS, Petkova L, Dzhenkov DL, Sapundzhiev NR, Todorov I. Gross and Histopathology of COVID-19 With First Histology Report of Olfactory Bulb Changes. Cureus. 2020; 12(12).

29. Bösmüller H, Traxler S, Bitzer M, Häberle H, Raiser W, Nann D, et al. The evolution of pulmonary pathology in fatal COVID-19 disease: an autopsy study with clinical correlation. Virchows Arch. 2020; 477(3): 349-57.

30. Scendoni. Histopath 2 oncology COVIDs. Diagn Pathol. 2020; $1-4$.

31. Bian X. Autopsy of COVID-19 patients in China. 2020 .

32. Aguiar D, Lobrinus JA, Schibler M, Fracasso T, Lardi C. Inside the lungs of COVID-19 disease. Int J Legal Med. 2020; 134(4): 1271-4.

33. Konopka KE, Wilson A, Myers JL. Postmortem Lung Findings in a Patient With Asthma and Coronavirus Disease 2019. Chest. 2020; 158(3): e99-101.

34. Chmielik E, Jazowiecka-Rakus J, Dyduch G, Nasierowska-Guttmejer A, Michalowski L, Sochanik A, et al. COVID-19 Autopsies: A Case Series from Poland. Pathobiology. 2020.

35. Ducloyer M, Gaborit B, Toquet C, Castain L, Bal A, Arrigoni PP, et al. Complete post-mortem data in a fatal case of COVID-19: clinical, radiological and pathological correlations. Int J Legal Med. 2020; 134(6): 2209-14.

36. Remmelink M, De Mendonça R, D’Haene N, De Clercq S, Verocq C, Lebrun L, et al. Unspecific postmortem findings despite multiorgan viral spread in COVID-19 patients. Crit Care. 2020; 24(1): 1-10.

37. Buja LM, Wolf D, Zhao B, Akkanti B, McDonald M, Lelenwa $\mathrm{L}$, et al. The emerging spectrum of cardiopulmonary pathology of the coronavirus disease 2019 (COVID-19): Report of 3 autopsies from Houston, Texas, and review of autopsy findings from other United States cities. Cardiovasc Pathol [Internet].
2020; 48: 107233. Disponible en: https://doi.org/10.1016/j.carpath.2020.107233

38. Sauter JL, Baine MK, Butnor KJ, Buonocore DJ, Chang JC, Jungbluth AA, et al. Insights into pathogenesis of fatal COVID-19 pneumonia from histopathology with immunohistochemical and viral RNA studies. Histopathology. 2020; 77(6): 915-25.

39. Calvin M, Cletus T, Madala N. Since January 2020 Elsevier has created a COVID-19 resource centre with free information in English and Mandarin on the novel coronavirus COVID-19. The COVID-19 resource centre is hosted on Elsevier Connect, the company's public news and information. 2020; (January).

40. Okudela K, Hayashi H, Yoshimura Y, Sasaki H, Horiuchi H, Miyata N, et al. A Japanese case of COVID-19: An autopsy report. Pathol Int. 2020; 70(10): 820-4.

41. Zeng Z, Xu L, Xie XY, Yan HL, Xie BJ, Xu WZ, et al. Pulmonary pathology of early-phase COVID-19 pneumonia in a patient with a benign lung lesion. Histopathology. 2020; 77(5): 823-31.

42. Flikweert AW, Grootenboers MJJH, Yick DCY, du Mée AWF, van der Meer NJM, Rettig TCD, et al. Late histopathologic characteristics of critically ill COVID19 patients: Different phenotypes without evidence of invasive aspergillosis, a case series. J Crit Care [Internet]. 2020; 59: 149-55. Disponible en: https://doi.org/10.1016/j.jcrc.2020.07.002

43. Rubin JE, Crowe SE. Annals of internal medicine ${ }^{\circledR}$. Ann Intern Med. 2020; 172(1): ITC1-14.

44. Beigmohammadi MT, Jahanbin B, Safaei M, Amoozadeh L, Khoshavi M, Mehrtash V, et al. Pathological Findings of Postmortem Biopsies From Lung, Heart, and Liver of 7 Deceased COVID-19 Patients. Int J Surg Pathol. 2020; 1-11.

45. Yao X, Li T, He Z, Ping Y, Liu H, Yu S, et al. Histopathological Study of New Coronavirus Pneumonia (COVID-19) in Three Patients. Chinese J Pathol. 2020; 49 DOI: 10.3760/cma.j.cn11215120200312-00193

46. Recalde-Zamacona B, García-Tobar L, Argueta A, Álvarez L, De Andrea CE, Fernández Alonso M, et al. 
Histopathological findings in fatal COVID-19 severe acute respiratory syndrome: Preliminary experience from a series of 10 Spanish patients. Thorax. 2020; 75(12): 1116-8.

47. Xu Z, Shi L, Wang Y et al. Pathological findings of COVID-19 associated with acute respiratory distress syndrome [published correction appears in Lancet Respir Med. 2020 Feb 25;:]. Lancet Respir Med. 2020; 8(4): 420-422. doi: 10.1016/S2213-2600(20)30076-X Lancet Respir Med [Internet]. 2020;8(feb 25):420-2. Disponible en: https://doi.org/10.1016/S2213$\underline{\text { 2600(20)30076-X }}$

48. Ding M, Zhang Q, Li Q, Wu T, Huang Y. Since January 2020 Elsevier has created a COVID-19 resource centre with free information in English and Mandarin on the novel coronavirus COVID-19. The COVID-19 resource centre is hosted on Elsevier Connect, the company's public news and information. 2020; (January).

49. Beasley MB, Franks TJ, Galvin JR, Gochuico B, Travis WD. Acute fibrinous and organizing pneumonia: A histologic pattern of lung injury and possible variant of diffuse alveolar damage. Arch Pathol Lab Med. 2002; 126(9): 1064-70.

50. Valdivia-Mazeyra MF, Salas C, Nieves-Alonso JM, Martín-Fragueiro L, Bárcena C, Muñoz-Hernández P, et al. Increased number of pulmonary megakaryocytes in COVID-19 patients with diffuse alveolar damage: an autopsy study with clinical correlation and review of the literature. Virchows Arch. 2020.

51. Schaefer IM, Padera RF, Solomon IH, Kanjilal S, Hammer MM, Hornick JL, et al. In situ detection of SARS-CoV-2 in lungs and airways of patients with COVID-19. Mod Pathol [Internet]. 2020; 33(11): 2104-14. Disponible en: http://dx.doi.org/10.1038/s41379-020-0595-Z

52. Barisione E, Grillo F, Ball L, Bianchi R, Grosso M, Morbini $\mathrm{P}$, et al. Fibrotic progression and radiologic correlation in matched lung samples from COVID-19 post-mortems. Virchows Arch. 2020.

53. Pernazza A, Mancini M, Rullo E, Bassi M, De Giacomo T, Rocca C Della, et al. Early histologic findings of pulmonary SARS-CoV-2 infection detected in a surgical specimen. Virchows Arch. 2020;
477(5): 743-8.

54. Elsoukkary SS, Mostyka M, Dillard A, Berman DR, Ma LX, Chadburn A, et al. Autopsy Findings in 32 Patients with COVID-19: A Single-Institution Experience. Pathobiology. 2020.

55. Sociedade Chinesa de Medicina de Reabilitação, Comitê de Reabilitação Respiratória da Associação Chinesa de Medicina de Reabilitação G de RC do R de MF e R da AMC, Qianxue M, Hao D, Yin Z, Hong Z, Hospital T. Nova Orientação para Reabilitação Respiratória de Coronavírus Pneumonia (Segunda Edição). 2020; 44: 1-8.

56. Mac Dowall MP, Barrionuevo-Poquet A, CarneroFuentes O, Pareja-Begazo G, Coayla-Cano C, GalloLopez A, et al. Clinical-pathological characterization, viral genotipification and genetic heterogeneity as risk determinants in COVID-19: Study design and initial findings. Rev la Fac Med Humana. 2020; 20(3): 43343.

57. Wang XX, Shao C, Huang XJ, Sun L, Meng LJ, Liu $\mathrm{H}$, et al. Histopathological features of multiorgan percutaneous tissue core biopsy in patients with COVID-19. J Clin Pathol. 2020; 1-6.

58. Eckermann M, Frohn J, Reichardt M, Osterhoff M, Sprung M, Westermeier F, et al. 3D Virtual Pathohistology of Lung Tissue From Covid-19 Patients Based on Phase Contrast X-Ray Tomography. Elife. 2020; 9: 1-25.

59. Kommoss FKF, Schwab C, Tavernar L, Schreck J, Wagner WL, Merle U, et al. Pathologie der schweren COVID-19-bedingten Lungenschädigung. Dtsch Arztebl Int. 2020; 117 (29-30): 500-6.

60.Ochoa Salmorán H, Martínez Martínez I, Díaz Greene EJ. Ventilación mecánica en pacientes con COVID-19 de acuerdo a los fenotipos de Gattinoni. Acta Médica Grup Ángeles. 2020; 18(3): 336-40.

61. Leng L, Cao R, Ma J, Mou D, Zhu Y, Li W, et al. Pathological features of COVID-19-associated lung injury: a preliminary proteomics report based on clinical samples. Signal Transduct Target Ther [Internet]. 2020; 5(1): 1-9. Disponible en: http://dx.doi.org/10.1038/s41392-020-00355-9 
62. COAGULOPATÍA EN PACIENTES CON COVID19 [Internet]. [citado 27 de enero de 2021]. Disponible en:

https://www.siicsalud.com/dato/resiiccompleto.php/1 $\underline{63612}$

63. Comino-Trinidad O, Calvo A, Ojeda A, Mercadal J, Cornellas L, Ferrando C. Disseminated intravascular coagulation as a form of presentation of coronavirus19 disease. Clinical case. Rev Esp Anestesiol Reanim. 1 de enero de $202 ; 68(1)$ : 41-5.

64. Iba T, Levy JH. Inflammation and thrombosis: roles of neutrophils, platelets and endothelial cells and their interactions in thrombus formation during sepsis. J Thromb Haemost. 2018; 16(2): 231-41.

65. Tang N, Li D, Wang X, Sun Z. Abnormal coagulation parameters are associated with poor prognosis in patients with novel coronavirus pneumonia. J Thromb Haemost [Internet]. 1 de abril de 2020 [citado 27 de enero de 2021]; 18(4): 844-7. Disponible en: /pmc/articles/PMC7166509/?report=abstract

66. Iba T, Levy JH, Levi M, Thachil J. Coagulopathy in COVID-19. J Thromb Haemost [Internet]. 21 de septiembre de 2020 [citado 27 de enero de 2021]; 18(9):2 103-9. Disponible en: https://onlinelibrary.wiley.com/doi/abs/10.1111/jth.14 $\underline{975}$

67. Giannis D, Ziogas IA, Gianni P. Coagulation disorders in coronavirus infected patients: COVID-19, SARS-CoV-1, MERS-CoV and lessons from the past. J Clin Virol. 1 de junio de 2020; 127: 104362. 